\title{
Electron-Electron Scattering In Single Quantum Well GaAs Laser With And Without Screening
}

\author{
Dr. Ragheed M. Ibrahim \\ Department of Physics \\ College of Education \\ University of Mosul
}

Received

$27 / 10 / 2010$

\author{
Dr. Erada A. Al-Dabbagh \\ Department of Physics \\ College of Science \\ University of Mosul
}

Accepted

18 / 01 / 2011

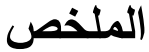

في هذا البحث تم دراسة معدل تتشت الكترون- الكترون في بئر الجهد الكمي الاحادي لا

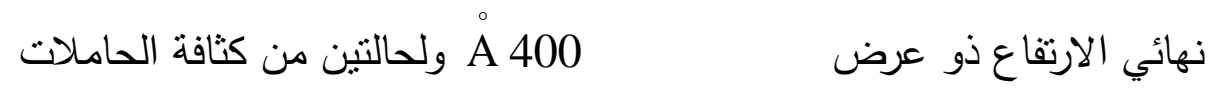

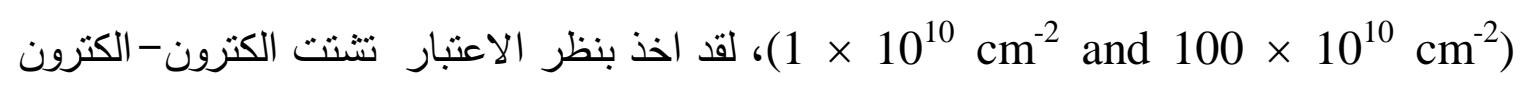

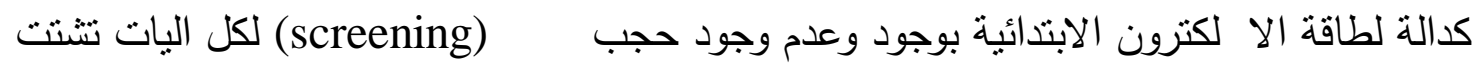
الكترون-الكترون في الحزم الفرعية.
\end{abstract}

\section{Abstract}

In this paper we have studied the electron-electron scattering rates in an infinitely deep single quantum well with width $(400 \mathrm{~A})$ and for both carrier densities $\left(1 \times 10^{10} \mathrm{~cm}^{-2}\right.$ and $\left.100 \times 10^{10} \mathrm{~cm}^{-2}\right)$, we have considered the electron-electron scattering as a function of the initial electron energy with and without screening at all subband carrier-carrier scattering mechanisms.

\section{Introduction}

Fermi's Golden Rule describes the lifetime of a particle in a particular state with respect to scattering by a time-varying potential. For phonon scattering, this harmonic potential is derived from the phonon wave function, which is itself a traveling wave. For the case of one carrier scattering against another due to the Coulomb potential, there appears to be no time dependency. The Born approximation is often cited in the literature 
when discussing carrier-carrier scattering (the transition of electron between two levels in the band); this is just a way of working scattering from a constant potential into Fermi's Golden Rule. This is achieved by considering that the perturbing potential is 'switched on' only when the particle reaches the same proximity. Therefore, the perturbating potential appearing in Fermi's Golden Rule for the interaction of two isolated carriers is the Coulombic interaction. Now the initial and final states, $|i\rangle$ and $|f\rangle$, respectively, of the system both consist of two electron (or hole) wave functions, as carrier-carrier scattering is a two-body problem, and thus there is a much greater variety of scattering mechanisms possible than in the essentially one-body problem encountered in phonon scattering ${ }^{[1,2]}$.

\section{Theory}

In a N-level system there are $\left(4^{\mathrm{N}}\right)^{[2]}$ different scattering events. In this two-level system these are as follows: 11-11, 11-12, 11-21, 11-22, 12-11, $12-12,12-21,12-22,21-11,21-12,21-21,21-22,22-11,22-12,22-21$, and 22-22. Note that completely different events of the type (ij-fj) are possible in quantum wells with three or more subbands, and interactions of this type have been shown to be important in optically pumped intersubband lasers $^{[3,4]}$.

the matrix element in Fermi's Golden Rule be expressed as by:

$\langle\mathbf{f}|\tilde{\mathcal{H}}| \mathbf{i}\rangle=\left\langle\psi_{\mathrm{f}}(z) \frac{\mathrm{e}^{-i \mathbf{k}_{\mathbf{f}} \mathbf{r}_{x y}}}{\sqrt{A}} \psi_{\mathrm{g}}\left(z^{\prime}\right) \frac{\mathrm{e}^{-i \mathbf{k}_{\mathbf{g}} \bullet \mathbf{r}_{x y}^{\prime}}}{\sqrt{A}}\left|\frac{e^{2}}{4 \pi \epsilon r}\right| \psi_{\mathrm{i}}(z) \frac{\mathrm{e}^{-i \mathbf{k}_{\mathbf{i}} \mathbf{r}_{x y}}}{\sqrt{A}} \psi_{\mathrm{j}}\left(z^{\prime}\right) \frac{\mathrm{e}^{-i \mathbf{k}_{\mathbf{j}} \bullet \mathbf{r}_{x y}^{\prime}}}{\sqrt{A}}\right\rangle$

where the subband indices of the initial states are labelled ' $i$ ' and ' $j$ ' and those of the final states ' $f$ ' and ' $g$ '. The decoupled form of the wave functions, with a component of the motion confined along the z-axis and an in-plane (x-y) travelling wave, suggests that the integrals should be evaluated across the plane and along the growth axis, and that the separation of the carriers be expressed as ${ }^{[\mathbf{5 , 6}, 7]}$

$$
r=\sqrt{\left|\mathbf{r}_{x y}-\mathbf{r}_{x y}^{\prime}\right|^{2}+\left(z-z^{\prime}\right)^{2}}
$$

Therefore obtain:

$$
\begin{aligned}
\langle\mathbf{f}|\tilde{\mathcal{H}}| \mathbf{i}\rangle & =\frac{e^{2}}{4 \pi \epsilon A^{2}} \int_{-\infty}^{+\infty} \int_{-\infty}^{+\infty} \iint \psi_{\mathbf{f}}^{*}(z) \psi_{\mathrm{g}}^{*}\left(z^{\prime}\right) \psi_{\mathrm{i}}(z) \psi_{\mathrm{j}}\left(z^{\prime}\right) \\
& \times \frac{\mathrm{e}^{-i\left(\mathbf{k}_{\mathbf{i} \bullet} \mathbf{r}_{x y}+\mathbf{k}_{\mathbf{j}} \mathbf{r}_{x y}^{\prime}\right)} \mathrm{e}^{i\left(\mathbf{k}_{\mathbf{f}} \mathbf{r}_{x y}+\mathbf{k}_{\mathrm{g}} \mathbf{r}_{x y}^{\prime}\right)}}{\sqrt{\left|\mathbf{r}_{x y}-\mathbf{r}_{x y}^{\prime}\right|^{2}+\left(z-z^{\prime}\right)^{2}}} d \mathbf{r}_{x y}^{\prime} \mathrm{d} \mathbf{r}_{x y} \mathrm{~d} z^{\prime} \mathrm{d} z
\end{aligned}
$$




\section{Dr. Ragheed M. Ibrahim \& Dr. Erada A. Al-Dabbagh}

followed earlier methods for carrier-carrier scattering in bulk and took the two-dimensional Fourier Transform of the Coulombic potential to give ${ }^{[8]}$

$$
\langle\mathrm{f}|\tilde{\mathcal{H}}| \mathbf{i}\rangle=\frac{2 \pi e^{2}}{4 \pi \epsilon A q_{x y}} A_{\mathrm{ijfg}}\left(q_{x y}\right) \delta\left(\mathbf{k}_{\mathrm{f}}+\mathbf{k}_{\mathrm{g}}-\mathbf{k}_{\mathrm{i}}-\mathbf{k}_{\mathrm{j}}\right)
$$

where $q_{x y}=\left|\mathbf{k}_{\mathbf{i}}-\mathbf{k}_{\mathbf{f}}\right|$, and $A_{\mathrm{ijfg}}$ is a form factor, i.e.

$$
A_{\mathrm{ijfg}}=\int_{-\infty}^{+\infty} \int_{-\infty}^{+\infty} \psi_{\mathrm{i}}(z) \psi_{\mathrm{j}}\left(z^{\prime}\right) \psi_{\mathrm{f}}^{*}(z) \psi_{\mathrm{g}}^{*}\left(z^{\prime}\right) \mathrm{e}^{-q_{x y}\left|z-z^{\prime}\right|} \mathrm{d} z^{\prime} \mathrm{d} z-(5)
$$

Using the form for the matrix element shown in equation (4) and then substituting directly into Fermi's Golden Rule gives the lifetime of a carrier in subband 'i' as follows:

$$
\frac{1}{\tau_{\mathrm{i}}}=\frac{2 \pi}{\hbar} \sum_{\mathrm{f}, \mathrm{g}}\left|\frac{2 \pi e^{2}}{4 \pi \epsilon A q_{x y}} A_{\mathrm{ijfg}}\left(q_{x y}\right)\right|^{2} \delta\left(\mathbf{k}_{\mathrm{f}}+\mathbf{k}_{\mathrm{g}}-\mathbf{k}_{\mathrm{i}}-\mathbf{k}_{\mathrm{j}}\right) \delta\left(E_{\mathrm{f}}^{\mathrm{t}}+E_{\mathrm{g}}^{\mathrm{t}}-E_{\mathrm{i}}^{\mathrm{t}}-E_{\mathrm{j}}^{\mathrm{t}}\right)
$$

Converting the summations over both final-state wave vectors into integrals introduces a factor of $L /(2 \pi)$ per dimension, thus giving a factor of $\mathrm{A}^{2} /(2 \pi)^{4}$ in total (where the general area $\mathrm{A}=\mathrm{L}^{2}$ ), therefore:

$$
\begin{aligned}
\frac{1}{\tau_{\mathrm{i}}}=\frac{2 \pi}{\hbar} \frac{A^{2}}{(2 \pi)^{4}} \iint & \left|\frac{2 \pi e^{2}}{4 \pi \epsilon A q_{x y}} A_{\mathrm{ijg}}\left(q_{x y}\right)\right|^{2} \delta\left(\mathbf{k}_{\mathrm{f}}+\mathbf{k}_{\mathrm{g}}-\mathbf{k}_{\mathrm{i}}-\mathbf{k}_{\mathrm{j}}\right) \\
& \times \delta\left(E_{\mathrm{f}}^{\mathrm{t}}+E_{\mathrm{g}}^{\mathrm{t}}-E_{\mathrm{i}}^{\mathrm{t}}-E_{\mathrm{j}}^{\mathrm{t}}\right) \mathrm{d} \mathbf{k}_{\mathrm{g}} \mathrm{d} \mathbf{k}_{\mathrm{f}}
\end{aligned}
$$

and thus:

$$
\begin{aligned}
\frac{1}{\tau_{\mathrm{i}}}=\frac{e^{4}}{2 \pi \hbar(4 \pi \epsilon)^{2}} & \iint \frac{\left|A_{\mathrm{ijfg}}\left(q_{x y}\right)\right|^{2}}{q_{x y}^{2}} \delta\left(\mathbf{k}_{\mathrm{f}}+\mathbf{k}_{\mathrm{g}}-\mathbf{k}_{\mathrm{i}}-\mathbf{k}_{\mathrm{j}}\right) \\
& \times \delta\left(E_{\mathrm{f}}^{\mathrm{t}}+E_{\mathrm{g}}^{\mathrm{t}}-E_{\mathrm{i}}^{\mathrm{t}}-E_{\mathrm{j}}^{\mathrm{t}}\right) \mathrm{d} \mathbf{k}_{\mathrm{g}} \mathrm{d} \mathbf{k}_{\mathrm{f}}
\end{aligned}
$$

Integrating over all of the states of the second carrier (given by $\mathrm{k}_{\mathrm{j}}$ ) and introducing Fermi-Dirac distribution functions to account for state occupancy, then obtain:

$$
\begin{aligned}
& \frac{1}{\tau_{\mathbf{i}}}=\frac{e^{4}}{2 \pi \hbar(4 \pi \epsilon)^{2}} \iiint \frac{\left|A_{\mathrm{ijfg}}\left(q_{x y}\right)\right|^{2}}{q_{x y}^{2}} f_{\mathrm{j}}^{\mathrm{FD}}\left(\mathbf{k}_{\mathrm{j}}\right)\left[1-f_{\mathrm{f}}^{\mathrm{FD}}\left(\mathbf{k}_{\mathrm{f}}\right)\right]\left[1-f_{\mathrm{g}}^{\mathrm{FD}}\left(\mathbf{k}_{\mathrm{g}}\right)\right] \\
& \times \delta\left(\mathbf{k}_{\mathrm{f}}+\mathbf{k}_{\mathrm{g}}-\mathbf{k}_{\mathrm{i}}-\mathbf{k}_{\mathrm{j}}\right) \delta\left(E_{\mathrm{f}}^{\mathrm{t}}+E_{\mathrm{g}}^{\mathrm{t}}-E_{\mathrm{i}}^{\mathrm{t}}-E_{\mathrm{j}}^{\mathrm{t}}\right) \mathrm{dk}_{\mathrm{g}} \mathrm{d} \mathbf{k}_{\mathrm{f}} \mathbf{d} \mathbf{k}_{\mathrm{j}}
\end{aligned}
$$


The first $\delta$-function summarizes in-plane momentum conservation and limits the integral over $\mathrm{k}_{\mathrm{g}}$ to a contribution when $\mathrm{k}_{\mathrm{g}}=\mathrm{k}_{\mathrm{i}}+\mathrm{k}_{\mathrm{j}}-\mathrm{k}_{\mathrm{f}}$. In addition, the total energy of the carriers, $\mathrm{E}_{\mathrm{i}}^{\mathrm{t}}$, etc. are equal to the energy of the relevant subband minima, $\mathrm{E}_{\mathrm{i}}$ say, plus the in-plane kinetic energy; thus ${ }^{[6]}$

$$
\begin{aligned}
\frac{1}{\tau_{\mathrm{i}}} & =\frac{e^{4}}{2 \pi \hbar(4 \pi \epsilon)^{2}} \iint \frac{\left|A_{\mathrm{ijfg}}\left(q_{x y}\right)\right|^{2}}{q_{x y}^{2}} P_{\mathrm{j}, \mathrm{f}, \mathrm{g}}\left(\mathbf{k}_{\mathrm{j}}, \mathbf{k}_{\mathrm{f}}, \mathbf{k}_{\mathrm{g}}\right) \\
& \times \delta\left(E_{\mathrm{f}}+\frac{\hbar^{2} \mathbf{k}_{\mathrm{f}}^{2}}{2 m^{*}}+E_{\mathrm{g}}+\frac{\hbar^{2} \mathbf{k}_{\mathrm{g}}^{2}}{2 m^{*}}-E_{\mathrm{i}}+\frac{\hbar^{2} \mathbf{k}_{\mathrm{i}}^{2}}{2 m^{*}}-E_{\mathrm{j}}+\frac{\hbar^{2} \mathbf{k}_{\mathrm{j}}^{2}}{2 m^{*}}\right) \mathrm{d} \mathbf{k}_{\mathrm{f}} \mathrm{d} \mathbf{k}_{\mathrm{j}--(10)}
\end{aligned}
$$

and therefore

$$
\begin{aligned}
\frac{1}{\tau_{\mathrm{i}}}= & \frac{m^{*} e^{4}}{\pi \hbar^{3}(4 \pi \epsilon)^{2}} \iint \frac{\left|A_{\mathrm{ijfg}}\left(q_{x y}\right)\right|^{2}}{q_{x y}^{2}} P_{\mathrm{j}, \mathrm{f}, \mathrm{g}}\left(\mathbf{k}_{\mathrm{j}}, \mathbf{k}_{\mathrm{f}}, \mathbf{k}_{\mathrm{g}}\right) \\
& \times \delta\left(\mathbf{k}_{\mathrm{f}}^{2}+\mathbf{k}_{\mathrm{g}}^{2}-\mathbf{k}_{\mathrm{i}}^{2}-\mathbf{k}_{\mathrm{j}}^{2}+\frac{2 m^{*}}{\hbar^{2}}\left(E_{\mathrm{f}}+E_{\mathrm{g}}-E_{\mathrm{i}}-E_{\mathrm{j}}\right)\right) \mathrm{d} \mathbf{k}_{\mathrm{f}} \mathrm{d} \mathbf{k}_{\mathrm{j}}
\end{aligned}
$$

where $\mathrm{k}_{\mathrm{g}}$ is known in terms of the other three wave vectors. It is the assumption of parabolic subbands in this last step that will be the limiting factor for the application of this method to hole-hole scattering-a point mentioned earlier in the context of the carrier-LO phonon scattering rate derivation. Now equation (11) represents the scattering rate of a carrier at a particular wave vector $\mathrm{k}_{\mathrm{i}}$ averaged over all of the other initial particle states $\mathrm{k}_{\mathrm{j}}$, and hence the only unknown in this remaining $\delta$-function is the wave vector $\mathrm{k}_{\mathrm{f}}$. Contributions to the integral over $\mathrm{k}_{\mathrm{f}}$ occur when the argument of the $\delta$-function is zero, and indeed given the form for this argument, it is clear that the solutions for $\mathrm{k}_{\mathrm{f}}$ map out an ellipse.

The standard procedures is to replace this integral over $\mathrm{k}_{\mathrm{f}}$ with one over an angle $\theta$ which describes the ellipse, thus giving:

$$
\frac{1}{\tau_{\mathrm{i}}}=\frac{m^{*} e^{4}}{4 \pi \hbar^{3}(4 \pi \epsilon)^{2}} \iint_{0}^{2 \pi} \frac{\left|A_{\mathrm{jjfg}}\left(q_{x y}\right)\right|^{2}}{q_{x y}^{2}} P_{\mathrm{j}, \mathrm{f}, \mathrm{g}}\left(\mathbf{k}_{\mathrm{j}}, \mathbf{k}_{\mathrm{f}}, \mathbf{k}_{\mathrm{g}}\right) \mathrm{d} \theta \mathrm{d} \mathbf{k}_{\mathrm{j}^{------}}
$$

but with an additional factor of 4 in the denominator. In this present treatise the factor $\mathrm{P}_{\mathrm{jfg}}\left(\mathrm{k}_{\mathrm{j}}, \mathrm{k}_{\mathrm{f}}, \mathrm{k}_{\mathrm{g}}\right)$ has been assumed to be dependent upon the initialstate distribution only, i.e. $\mathrm{P}_{\mathrm{jfg}}\left(\mathrm{k}_{\mathrm{j}}, \mathrm{k}_{\mathrm{f}}, \mathrm{k}_{\mathrm{g}}\right)=f_{\mathrm{j}}^{\mathrm{FD}}\left(\mathrm{k}_{\mathrm{j}}\right)$, and $\mathrm{q}_{x y}$ is given by equation

$$
\left(2 q_{x y}\right)^{2}=2 k_{\mathrm{ij}}^{2}+\Delta k_{0}^{2}-2 k_{\mathrm{ij}} \sqrt{k_{\mathrm{ij}}^{2}+\Delta k_{0}^{2}} \cos \theta
$$




\section{Dr. Ragheed M. Ibrahim \& Dr. Erada A. Al-Dabbagh}

The integration over the vector $\mathrm{k}_{\mathrm{j}}$ can be performed by effectively switching to plane polar coordinates, and integrating along the length $\mathrm{k}_{\mathrm{ij}}$ and around the angle a between $k_{i}$ and $k_{j}$. With this change in the integral, equation (12) then becomes ${ }^{[5,6]}$

$$
\frac{1}{\tau_{\mathrm{i}}}=\frac{m^{*} e^{4}}{4 \pi \hbar^{3}(4 \pi \epsilon)^{2}} \iint_{0}^{2 \pi} \int_{0}^{2 \pi} \frac{\left|A_{\mathrm{ijg}}\left(q_{x y}\right)\right|^{2}}{q_{x y}^{2}} P_{\mathrm{j}}\left(\mathbf{k}_{\mathrm{j}}\right) \mathrm{d} \theta \mathrm{d} \alpha k_{\mathrm{j}} \mathrm{d} k_{\mathrm{j}}
$$

So far, the theoretical treatise has only considered carrier-carrier scattering of two carriers in an empty environment. In semiconductor heterostructures, carrier-carrier scattering occurs because the system is doped, and therefore by definition there will be many carriers. In such instances, the force between any two carriers is not just the bare Coulombic repulsion, as the other mobile carriers are able to respond to any change in the electrostatic field, with the result being a reduction in the probability of scattering; the other carriers are said to screen the interaction. One of the simplest models for screening considers only the carriers within the same subband as the initial carrier state, it then proceeds by replacing the dielectric constant $\epsilon$ with one which is dependent upon the relative wave vector $q_{x y}$, i.e.

$$
\epsilon_{\mathrm{sc}}=1+\frac{2 \pi e^{2}}{(4 \pi \epsilon) q_{x y}} \Pi_{\mathrm{ii}}\left(q_{x y}\right) A_{\mathrm{ijg}}\left(q_{x y}\right)
$$

Where $\prod_{\mathrm{ii}}\left(q_{\chi y}\right)$ is the polarization factor and $A_{i j f g}$ is the form factor.

\section{Results and Discussion}

We consider the single quantum well, in an infinitely deep, and (400 ̊) wide GaAs quantum well at temperature $\left(70 \mathrm{~K}^{\circ}\right)$ for booth carrier density $\left(1 \times 10^{10} \mathrm{~cm}^{-2}\right.$ and $\left.100 \times 10^{10} \mathrm{~cm}^{-2}\right)$ in each level. We was adopted the same model of P. Harrison in our calculation to make it as a mailstone in our study ${ }^{[2]}$, he include in this calculation only (22-11) electron-electron scattering rate. In this work all kinds of scattering (intersubband $\&$ intrasubband) were taken into account, i.e. all the $16^{\text {th }}$ carrier- carrier scattering rates.

Two groups of scattering rates can be considered. Group (I), whose scattering rate decreases with increasing energy of the initial electron in state $|i\rangle$. Group (II) with its scattering rate increases with increasing energy. All kinds of scattering rates in group (II) are increases with increased energy. We noticed that the amount of scattering rates are too small 
$\left(10^{-18}-10^{-20} \mathrm{sec}^{-1}\right)$, i.e. the life time of carriers in initial state are of order $\left(10^{18}-10^{20} \mathrm{sec}\right)$-peculiarity- thus they have been neglected since they don't contribute in scattering ${ }^{[3]}$. All kinds of scattering rate in group (I) are the intrasubband scattering (where the number of carriers in each subband does not changes $)^{[4]}$ except the (22-11) scattering rate which is intersubband scattering (where at least one of the carriers changes its subband) ${ }^{[4]}$.

Figs. (1) and (2) show the electron-electron scattering rates with two levels of carrier density $\left(1 \times 10^{10} \mathrm{~cm}^{-2}\right.$ and $\left.100 \times 10^{10} \mathrm{~cm}^{-2}\right)$ with and without screening. The effect of screening on the scattering rate behaviour will be discussed later, now we just discuss the scattering behaviour without screening effect.

For $\left(\mathrm{N}=1 \times 10^{10} \mathrm{~cm}^{-2}\right)$, all shapes in Fig. (1) show a good behaviour of scattering rate i.e. the electron-electron scattering decreases with increasing of energy in initial level |i $\rangle$, that also means the more carriers were accumulated in the initial state with increasing energy level, and this what we expected. This can be generalized in group (I) except Fig.(1-b) at low energy, and since Fig.(1-a) and Fig.(1-b) have the same range of scattering rates, we make a comparison between them. At low, energy, the two kinds of scattering (22-11) and (11-22) behave oppositely, this means they occur respectively until reaching energy equal approximately to $\left(1 \times 10^{2} \mathrm{meV}\right)$, they would have the same shape and begin to be have normally.

For $\left(\mathrm{N}=100 \times 10^{10} \mathrm{~cm}^{-2}\right)$, the scattering rates are near by two orders of magnitude high in the higher-carrier-density case for (22-11) and (11-22) scattering rate as show in Fig.(2-a) and Fig.(2-b). The effect of high carrier density is more less for $(11-11,12-12,21-21,22-22)$ scattering rates as shown in Figs.(1-c,1-d,1-e,1-f) and Figs.(2-c,2-d,2-e,2-f), the scattering rates secondly increase for (12-21) and (21-12) and they have same scattering rates, which means they occur simultaneously.

In the addition of screening and for $\left(\mathrm{N}=1 \times 10^{10} \mathrm{~cm}^{-2}\right)$, the effect is smaller at low carrier density and just for one kind of scattering (22-11), as shown in Fig.(1-a), while there is no effect of screening on the scattering raters for other kinds of scattering as show in Fig. (1-b,1-c,1-d,1-e,1-f,1-g,1-h).

The effect of screening is a obvious clearly at higher carrier density $\left(\mathrm{N}=100 \times 10^{10} \mathrm{~cm}^{-2}\right)$ and for (22-11) and (11-22) scattering rates as shown in Fig.(2-a,2-b), and there is no effect for other kinds of scattering. So, it is apparent that screening reduces the scattering rates, and the higher the carrier density, then the larger the effect of screening, as might be expected. 
Dr. Ragheed M. Ibrahim \& Dr. Erada A. Al-Dabbagh
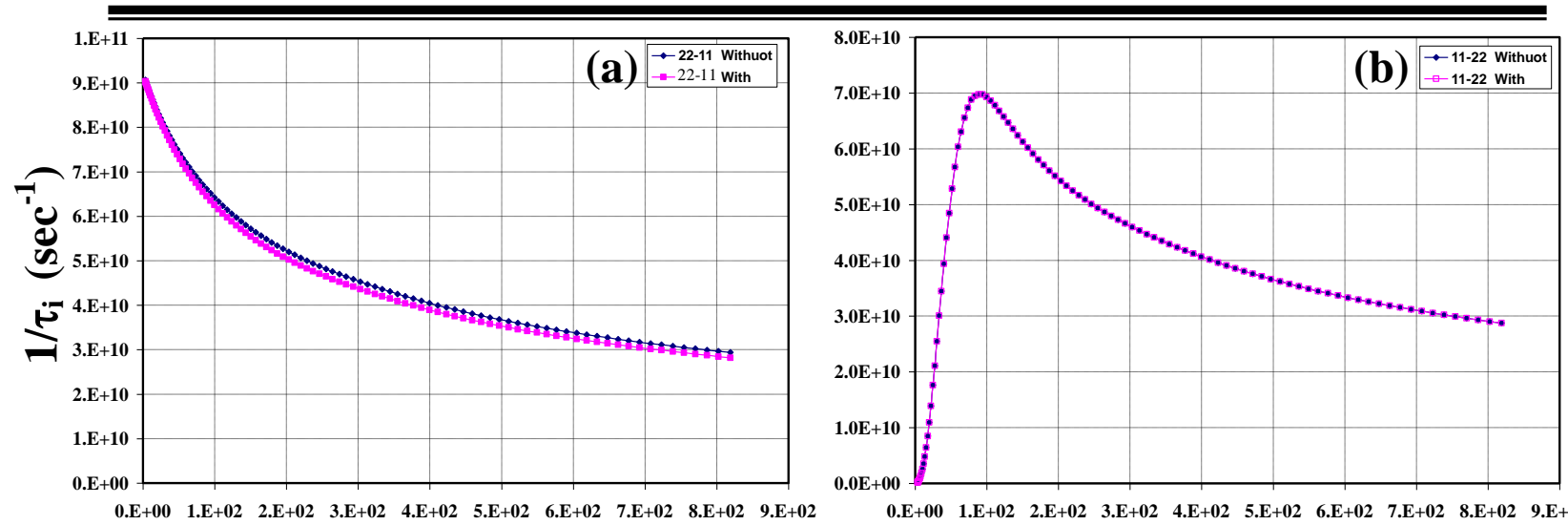

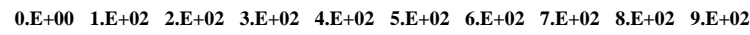
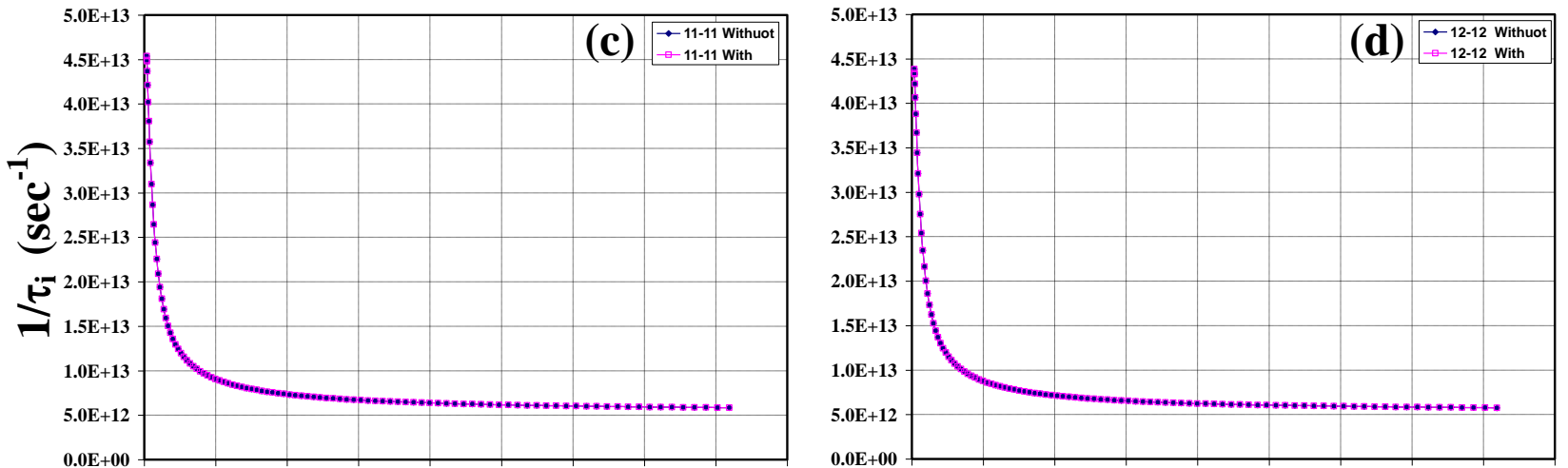

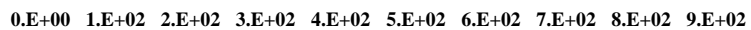

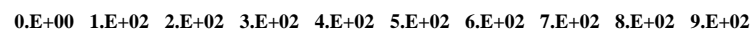
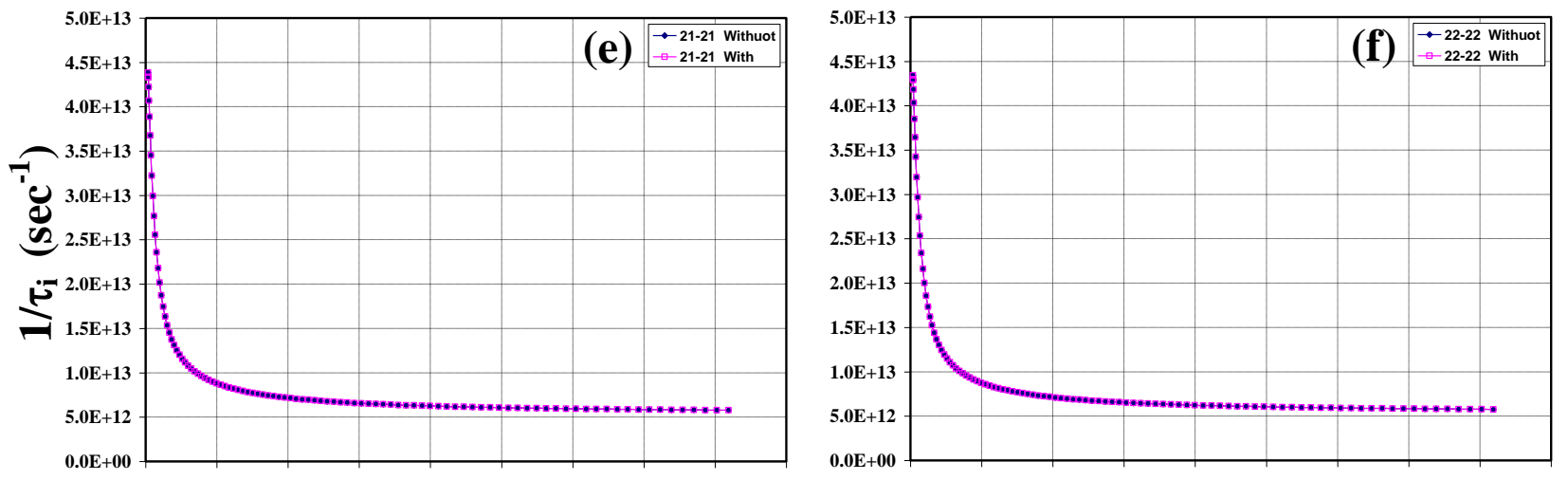

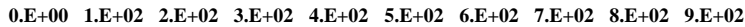

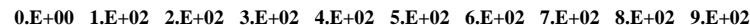

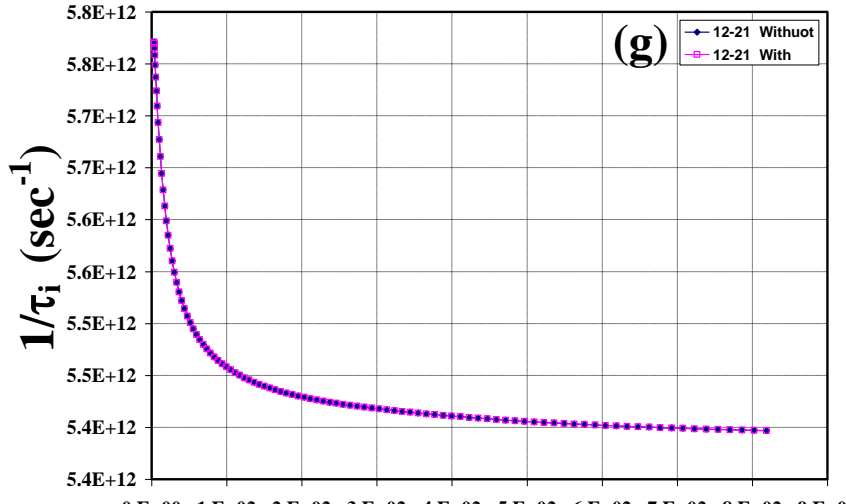

0.E+00 1.E+02 2.E+02 3.E+02 4.E+02 5.E+02 6.E+02 7.E+02 8.E+02 9.E+02

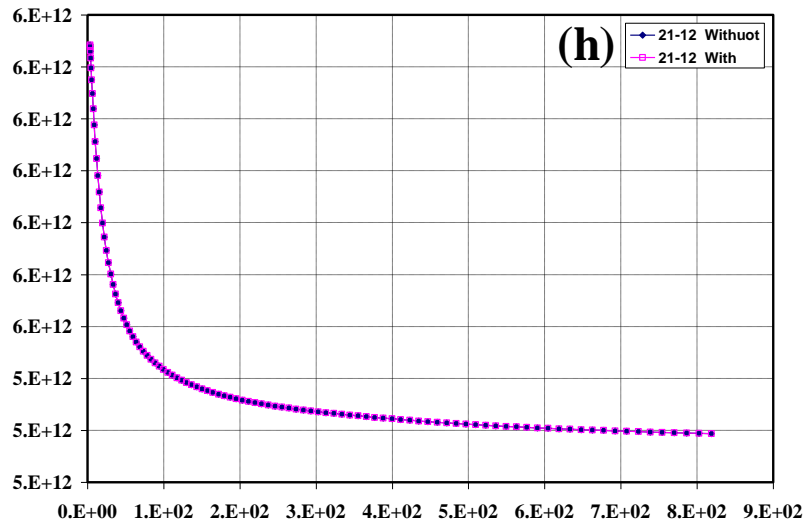

Initial Electron Energy (meV)

Fig.(1): The electron-electron scattering rate as a function of the initial electron energy, with and without screening, for carrier density $1 \times 10^{10} \mathrm{~cm}^{-2}$ 

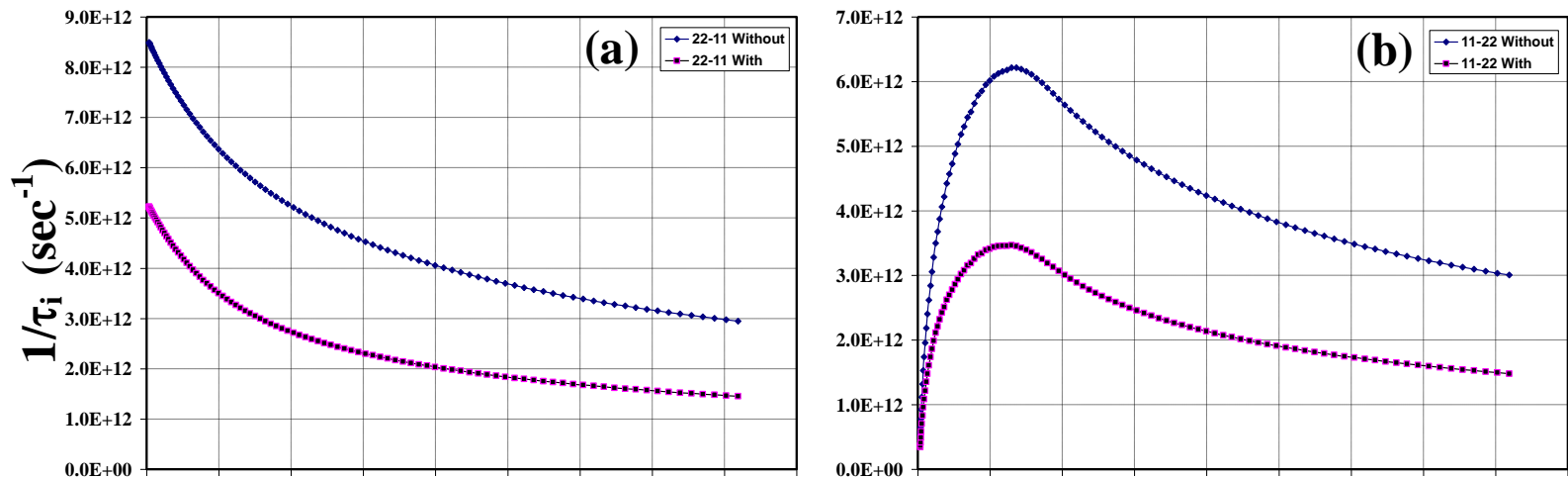

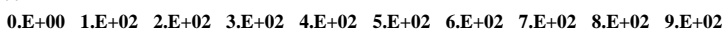

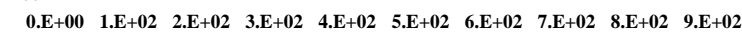
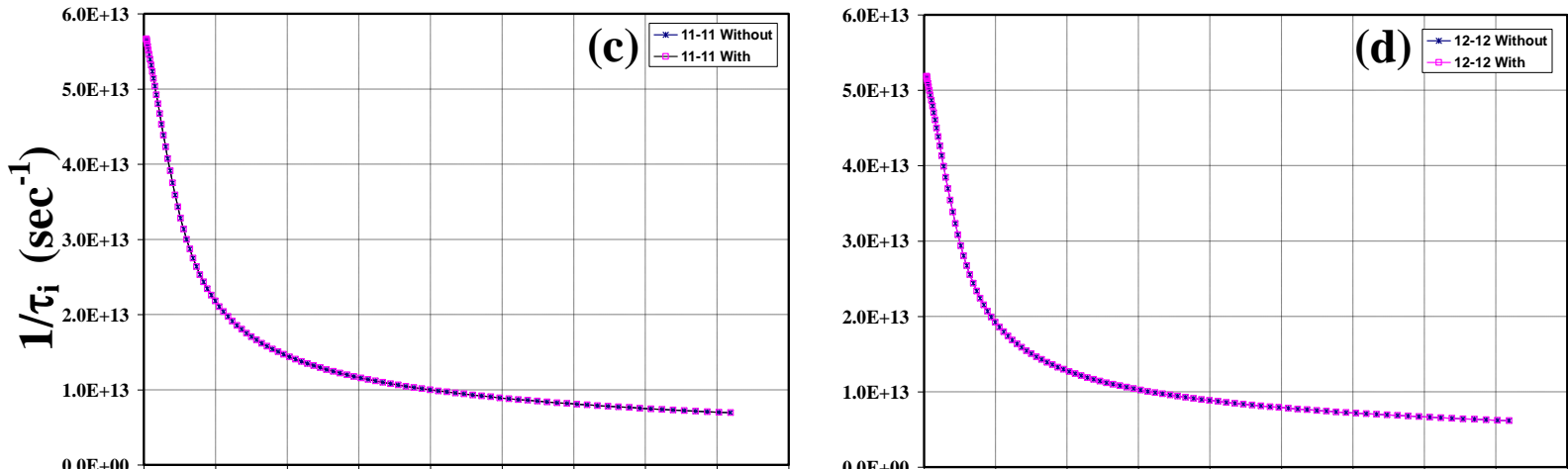

0.E+00 1.E+02 2.E+02 3.E+02 4.E+02 5.E+02 6.E+02 7.E+02 8.E+02 9.E+02

0.E+00 1.E+02 2.E+02 3.E+02 4.E+02 5.E+02 6.E+02 7.E+02 8.E+02 9.E+02
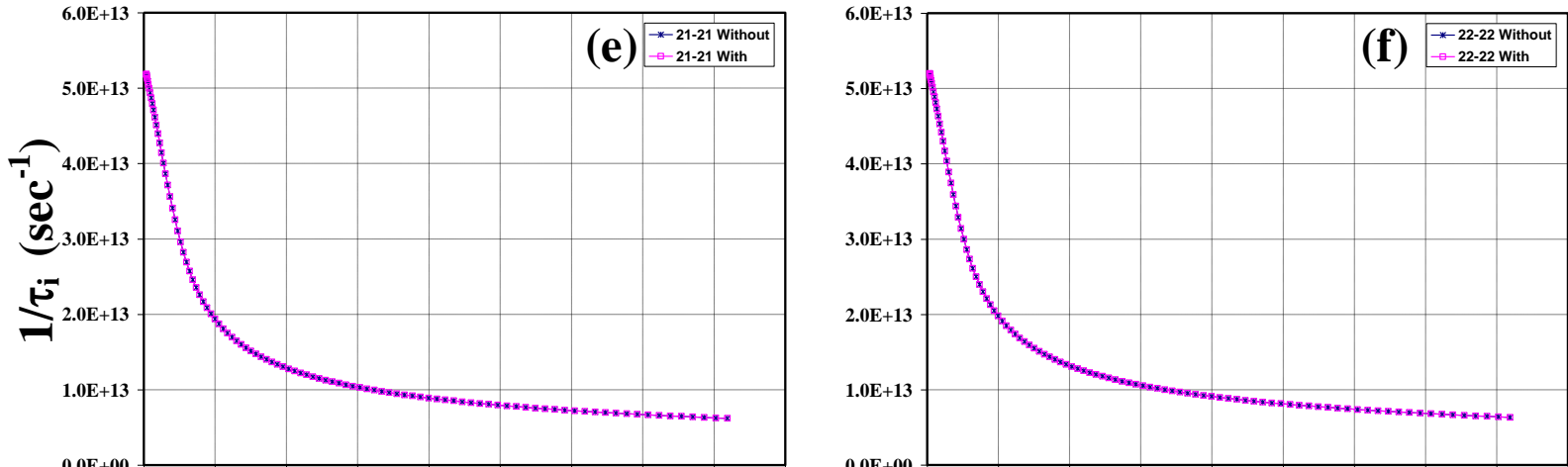

0.E+00 1.E+02 2.E+02 3.E+02 4.E+02 5.E+02 6.E+02 7.E+02 8.E+02 9.E+02

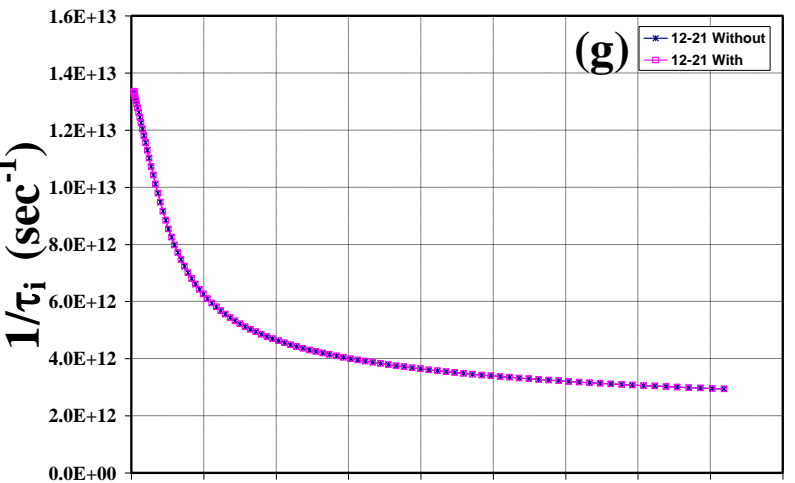

$0 . \mathrm{E}+00 \quad$ 1.E+02 2.E+02 3.E+02 4.E+02 5.E+02 6.E+02 7.E+02 8.E+02 9.E+02

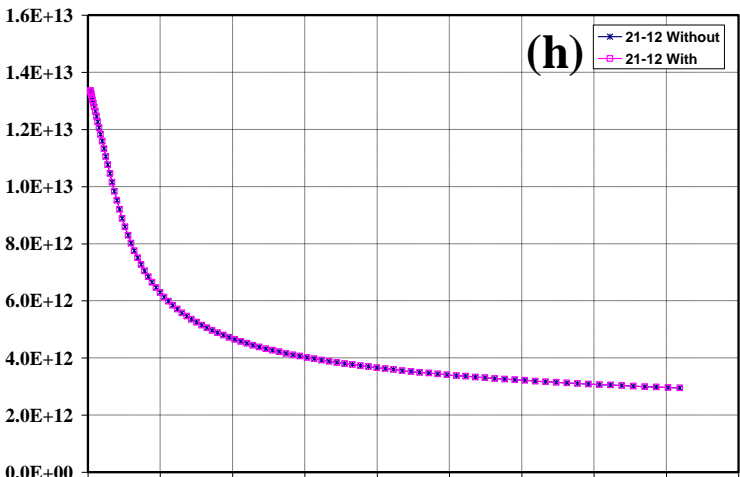

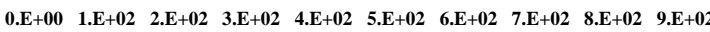

Initial Electron Energy

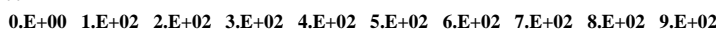

Initial Electron Energy

Fig.(2): The electron-electron scattering rate as a function of the initial electron energy, with and without screening, for carrier density $100 \times 10^{10} \mathrm{~cm}^{-2}$ 


\section{Dr. Ragheed M. Ibrahim \& Dr. Erada A. Al-Dabbagh}

\section{Conclusion}

Two group of scattering rates were studied for their behaviour under the effect of carrier densities and screening. Group two was ignored due to its strange behaviour. The scattering rate was increased as carrier density increasing for (22-11) and (11-22) scattering rate, which is more than the other kinds of scattering.

The effect of screening on the scattering rate for low carrier density $\left(1 \times 10^{10} \mathrm{~cm}^{-2}\right)$ is very small as if they were not exist, while it was obvious for higher density $\left(100 \times 10^{10} \mathrm{~cm}^{-2}\right)$ specially for (22-11) and (11-22) scattering.

\section{References}

1. P. Harrison, P. Kinsler, K. Donovan, and R. W. Kelsall, "The carrier dynamics of intersubband terahertz lasers" unpublished, available at URL:http://eenweb.leeds.ac.uk/homes/PH/projects/qcl/, 2007.

2. P. Harrison 'Quantum Wells, Wires and Dots' John Wiley \& Sons Ltd, Second Edition, 2005.

3. P. Kinsler, P. Harrison, and R. W. Kelsall, 'Intersubband electronelectron scattering in asymmetric quantum wells designed for farinfrared emission', Phys. Rev. B, 58:4771- 4778,1998.

4. S. C. Lee and I. Galbraith, "Intersubband and intrasubband electronic scattering rates in semiconductor quantum wells," Physical Review B, vol. 59, no. 24, pp.15796-15805, 1999.

5. S. M. Goodnick and P. Lugli, 'Effect of electron-electron scattering on non-equilibrium transport in quantum well systems', Phys. Rev. B, 37:2578, 1988.

6. J. H. Smet, C. G. Fonstad, and Q. Hu, 'Intrawell and interwell intersubband transitions in multiple quantum wells for far-infrared sources', J. Appl. Phys., 79:9305, 1996.

7. C. Peschke, "The impact of electron-electron interaction on electron transport in GaAs at high electric fields," Journal of Physics: Condensed Matter, vol. 6, no. 35, pp. 7027-7044, 1994

8. N. Takenaka, M. Inoue, and Y. Inuishi, 'Influence of inter-carrier scattering on hot electron distribution function in GaAs', J. Phys. Soc. Japan, 47:861,1979. 